\title{
Foundations of Organizational Structures in Multiagent Systems
}

\author{
Davide Grossi, Frank Dignum, Mehdi Dastani \\ Utrecht University, \\ The Netherlands \\ \{davide,jj,dignum\}@cs.uu.nl \\ Lambèr Royakkers \\ Eindhoven University of Technology \\ The Netherlands \\ l.m.m.royakkers@tm.tue.nl
}

\begin{abstract}
We analyze the notion of organizational structure in multiagent systems and explain the precise added value and the effects of such organizational structure on the involved agents. To pursue this aim, contributions from social and organization theory are considered which provide a solid theoretical foundation to this analysis. We argue that organizational structures should be seen along at least three dimensions, instead of just one: power, coordination, and control. In order to systematize the approach, formal tools are used to describe the organizational structure as well as the effect of such structures on the activities in multiagent systems. We specify the properties and the consequences of organizational structures for the actions of the involved agents.
\end{abstract}

\section{Introduction}

Many methodologies for multiagent systems (MAS) are based on organizational structures as their cornerstones. The organizational structure of MAS involves two basic concepts: agent roles and their relations in terms of which the collective behavior of individual agents is specified and the overall behavior of the MAS is determined. The specification of the overall behavior of MAS concerns the optimization of agents' activities, the management and security of the information flow among agents, and the enforcement of certain outcomes. Agent roles and their relations are often described by a variety of social concepts and relations like norm, power, delegation of tasks, responsibilities, permissions, access to resources, and communication.

The relations between agent roles can impose certain organizational structures on the roles that are crucial for the organization of MAS. A typical abstract example of 
such structures is the so-called "vertical differentiation" or "authority structure" of organizations, usually considered to be a "hierarchy" structure. These abstract types of structures are traditionally studied in the branch of sociology called mathematical soci$\operatorname{ology}([12,26,11])$. In fact, what was the case up to the sixties in literature on sociology and organization theory, is now being the case in the literature about organizations in MAS.

"The word "structure" is found extensively in the literature of the social sciences. "Social structure" and such related concepts such as "kinship structure", "authority structure", "communication structure", and "sociometric structure" are commonplace. [...] But despite the widespread use of structural concepts in the social sciences, it is fair to say that the formal analysis of structure has been relatively underdeveloped in these fields. The technical terminology employed in describing structures is meager; few concepts are defined rigorously. As a consequence, the social scientific description of structural properties tends to be couched in ambiguous terminology, and detailed studies of structure, as such, are rather rare." [17]

In the literature of MAS, these abstract types of organizational structures are studied and described only in an informal way, e.g. through pictures depicting tree-like configurations of points and lines like in [7]. In such informal studies, many issues remain hidden behind these pictures. Is the authority relation transitive, so that if $\mathrm{A}$ has authority over B and B over C then A has authority over C? Is it antisymmetric, so that if A has authority on B and B has authority on A then A and B are actually the same? However, if we want the notion of structure to be of any practical use for implemented MAS, pictures are plainly not enough, since they do not state with the necessary precision what are the properties of the organization that is described by the structure.

In this paper we will import notions from sociology and organization theory to describe a more rigorous foundation of organizational structures in MAS, which will be informally exposed in Section 2. In order to describe organizational structures we have to first describe exactly what the meaning is of the relations that form the structure. E.g. what is the meaning of a "power" relation and, maybe even more importantly, what are the consequences of the existence of such a relation between two agents? We will introduce a modal logic for this characterization in Section 3. In Section 4 we will discuss some properties of the relations and also give some intuitions about some natural properties of the organizational structure(s) that follow from them. Most importantly, we show that there are several organizational structures to be found and that the interplay between these structures for a large part determines the effective functioning of the organization. Finally, in Section 5, we draw some conclusions and we indicate some research lines worth pursuing in future work. 


\section{Organizational Structure}

Organizations "represent rationally ordered instruments for the achievement of stated goals" ([25]), that is, organizations arise in order to achieve specific objectives, and these objectives are pursued defining a number of subgoals contributing to the overall purpose of the organization. These subgoals identify the roles that are played in the organization. The relation between subgoals and overall objectives of the organization, i.e., the primitive decomposition of tasks within the organization, defines the essential form of organizational structure: "viewed in this light, formal organization is the structural expression of rational action" [25]. Roles are the basic units over which this structure ranges determining the source of the "rational order" holding in the organization. The above quotes consider then the decomposition of tasks as the central source of structure within organizations: structure is necessary for each organization to pursue its objectives.

Work on organization in $\mathrm{MAS}^{1}$ presents organizational structure as something essentially mono-dimensional, though it often, but only implicitly, considers a multiplicity of structured aspects: "authority", "communication", "delegation", "responsibility", "control", "decision-making", "power", etc. The thesis we hold here, which is inspired by foundational work on social and organization theory like [25, 23, 14], is that organizations do not exhibit one single structural dimension, but that they are instead multi-structured objects. In particular, we view organizational structure as hiding at least three relevant dimensions which we call: power, coordination and control. We will analyze power in relation with the delegation activity, coordination in relation with the knowledge and information issues, and control in relation with the monitoring and recovery issues. As a result of this analysis, organizations will be represented as explicitly displaying a triple structure constrained on the basis of the interplay between the three notions of power, coordination, and control. This structure, which is based on goal or task decomposition, relates then the roles of the organization ordering them with respect to the three aforementioned dimensions. Although we do not pretend to give full definitions of these relations (see $[3,21]$ for some more elaborate definitions of the delegation and power relations) we will characterize these relations in terms of some of their consequences for the agents enacting the roles between which these relations are defined.

Following work presented in $[4,10,5,6]$, roles are conceived here in terms of three basic notions: objectives, norms and information. As we will see, we will make use of these notions in order to isolate a few characteristics of the organizational structures given above.

Objectives constitute the essential component of roles, specifying to what the agents commit when they enact a role (objectives of the role become goals of the enacting agent). Norms specify instead, for each role, what are the constraints (essentially concerning interaction) which the organization imposes on actors playing that role. The distribution of objectives to roles implements a specific decomposition of tasks according to which the organization is supposed to function. What characterizes organizations though, is the possibility for the agents enacting the roles to delegate some of

\footnotetext{
${ }^{1}$ See [19] for an exhaustive survey.
} 
their goals, which come from the objectives of the roles they enact, to other agents. Delegation consists thus in the possibility for an agent enacting a role to transfer a given goal to a somehow subordinated one. This transfer takes place in the form of a directed obligation ([8]) of the agent enacting the first role to the agent enacting the subordinated one. This second agent is thus obliged to achieve a goal which belonged to the first agent, and therefore to include it in its own goal base. The possibility of delegating goals constitutes one of the essential aspects displaying what is usually called "delegation" or "power" structure of an organization ([20]): who delegates to whom?

Objectives and norms also determine to a great extent the control structure. Since objectives can be achieved or not, and norms can be respected or violated, control is an indispensable activity in which any organization has to engage. This is the case also for MAS organizations where agents, even if "benevolent", are anyway subjected to the possibility of failure. In its simplest form, control consists in a monitoring activity triggering appropriate reactions to determinate failures or violations. If an agent fails in achieving one of the stated or delegated objectives, a kind of supervisor agent should engage in the achievement of that objective: organization calls for a form of supervision activity ([14]). Potentially, the achievement of any objective as well as the compliance to any norm can be object of control. Because of this, control can be seen as "an organization within an organization" ([23]). With respect to control the relevant structural question is: who controls whom?

Objectives and norms are also coupled to the coordination structure, a broadly investigated topic in MAS studies. However, following $[7,15]$ we adopt here a simple view on coordination, reducing it to the issue of the information with which agents enacting specific roles should be endowed in order to achieve their goals and to abide by the norms of the organization.

Therefore, besides objectives and norms, roles should contain also the information necessary for agents to enact them. This turns into a knowledge problem of the state of the organization (or of part of $\mathrm{it}^{2}$ ) at a given moment. Agents should know when to act, that is, they should be informed about the status of the activities of the organization on which their activities depend ${ }^{3}$, and what they are obliged to do. As we observed above, delegation introduces a dynamics in the task distribution of an organization and in the set of norms effective in it. The point is that once a task is delegated and a correspondent obligation arises for a specific agent, a certain amount of information might be required for that agent to include that task in its own goal base and to pursue it. Because of this, an information mechanism which can keep track of this dynamics is crucial for the performance of an organization. To quote [23]:

The description of a delegation system [delegation structure] is incomplete unless the simultaneous signaling system [information structure] applied to it is also explicitly described.

\footnotetext{
${ }^{2}$ Notice, in passing, that the amount of knowledge to be propagated through the organization also constitutes an important issue:

If every competence [role] had full information about every other it might help but not necessarily; it would clearly be wasteful, if not physically impossible, for most organizations [23].

${ }^{3}$ This issue has been formally investigated in [15].
} 
The "information structure" should then guarantee that each agent has a representation of the actual state of the organization (both its objectives and norms as well as its operational state) which is sufficient for it to properly enact its role. The question is then how the access and sharing of information is structured within the organization: who informs whom?

Besides objectives, norms and information, the capabilities of a role are also an issue worth mentioning, though we will not consider it in detail here. It is somehow analogous to the information issue since it concerns what is presupposed by each role in order to achieve the relevant goals and comply with the relevant norms. A basic type of capabilities lies in the amount of resources that agents should have at their disposal. A second kind of capabilities play a central role in organizations, namely those concerning the so-called institutional power $([21,2])$. Again the problem is related with the dynamics introduced by the "delegation structure": delegating a task may require a parallel enabling or empowering activity such as making the relevant resources accessible, e.g., electronic money, and providing the required form of institutional empowerment, e.g., a suitable document. In this case the relevant structural question is: who enables or empowers whom? In order not to complicate matters further we assume in this paper that all agents have the capabilities needed to enact the role they fulfill, leaving this issue to future work.

\section{A Logic of Organization}

To describe an organization and its structure we will use a (typed) multi-modal propositional logic. As explained in the previous sections, we are interested in describing organizational structures and in reasoning about their consequences on the activities of the agents involved in the organization. To this aim, we propose a framework consisting of two basic components. The first component is geared toward representing finite relational configurations, that is the organizational structure ranging on agents, in propositional logic via special propositions. The second component handles instead agents' obligations, knowledge, goals and actions via a multi-modal logic.

The organizational structures are described making use of the special propositions Power $(r, s)$ to indicate that 'the agent enacting role $r$ has the agent enacting role $s$ in its power' (i.e. the agent playing role $r$ can delegate goals to the agent playing role $s$, Coordination $(r, s)$ to indicate that 'the agent enacting role $s$ has access to the information that is accessible to the agent enacting role $r$ ', and $\operatorname{Control}(r, s)$ to indicate that 'the agent enacting role $r$ controls the agent enacting role $s$ ' (in some sense, the agent playing role $r$ is "responsible" for the agent playing role $s$ ). Note that these propositions encode relations which are intended to hold between roles. We denote the fact that agent $a$ enacts role $r$, i.e., is a role enacting agent ([9]), by the special proposition rea $(a, r)$. In order to represent the norms, objectives and knowledge of a role in an organization, we make use of special propositions of respectively the form: $\operatorname{norm}(r, \phi), o b j(r, \phi)$ and $\inf (r, \phi)$, where $\phi$ is a propositional formula and $r$ a role.

Furthermore, in order to express the effects of organizational structure on the agents enacting the roles of the organization, we use a modal operator $K_{a}$ for representing the knowledge accessible to an agent $a, G_{a}$ for the goals that are pursued by the agent 
$a$, and $O_{a}$ for the obligations assigned to agent $a$. For the characterization of agents' activities we build on dynamic logic ([18]). Briefly, dynamic logic consists of the normal propositional language, extended with modal operators $[\alpha]$ for every action $\alpha$ in the language. These actions are either atomic (primitive) or composed by means of operators. In this work, only the atomic fragment of dynamic logic will be used, that is to say, no characterization of composite actions is used. An expression $[\alpha] \phi$ is read as 'the performance (execution) of the action $\alpha$ leads necessarily to a state (possible world) in which $\phi$ holds'. The formal semantics is given by means of a Kripke structure where there are accessibility relations $R_{\alpha}$ associated with each action $\alpha$. In this paper we will consider a set of action expressions consisting of at least all action expressions of the form delegate $(a, b, \phi)$ (meaning that $a$ delegates task $\phi$ to $b$ ); inform $(a, b, \phi)$ (meaning that $a$ informs $b$ about the state description $\phi$ ); and monitor $(a, D O N E(\alpha(b))$ ) (meaning that $a$ monitors the performance of action $\alpha$ by b).

We can now give a full formal definition of the syntax of our description language Org:

Definition 1 ( Syntax of $O r g$ )

Given a finite set $A R$ of role names, a finite set Ag of agent names, a countable set $P_{0}$ of atomic propositions from which the standard propositional language $L_{0}$ is built, a finite set of parameterized actions $\mathcal{A}$ (in general the elements of $\mathcal{A}$ are denoted by $\alpha(a)$ with $a \in A g$ representing the agent executing $\alpha)$ which contains at least the set of expressions $\{$ delegate $(a, b, \phi)$, inform $(a, b, \phi)$, monitor $(a, D O N E(\alpha(b))) \mid a, b \in A g$ and $\alpha(b) \in \mathcal{A}\}$, a set $P_{r}=\left\{\operatorname{norm}(r, \phi), \operatorname{obj}(r, \phi)\right.$, inf $\left.(r, \phi) \mid r \in A R, \phi \in L_{0}\right\}$ of specific propositions concerning the norms, objectives and information of roles, and a set $P_{s}=\{\operatorname{Power}(r, s)$, Coordination $(r, s)$, Control $(r, s)$, rea $(a, r) \mid r, s \in A R, a \in$ $A g\}$ of specific propositions concerning structural configurations, the admissible formulas of the Org language are recursively defined as follows:

- $P_{0} \cup P_{r} \cup P_{s} \subseteq O r g$

- If $\phi$ and $\psi \in$ Org, then $\phi \wedge \psi, \neg \phi \in O r g$

- If $\phi \in O r g$ and $a, b \in A g$, then $K_{a}(\phi), G_{a}(\phi), O_{a}(\phi) \in O r g$

- If $\phi \in \operatorname{Org}$ and $\alpha(a) \in \mathcal{A}$, then $[\alpha(a)] \phi \in O r g$

- If $\alpha(a) \in \mathcal{A}$, then DONE $(\alpha(a)) \in$ Org

Binary connectives $\rightarrow$ and $\vee$, and nullary connective $\perp$ can be defined as usual.

The semantics of the language $O r g$ will be given in two steps. First we define the tuples which constitute our formal modeling of the notion of an organizational structure (Definition 2). Secondly, these tuples will be used to give an explicit semantics to all special propositions of the form $\operatorname{Power}(r, s), \operatorname{Coordination}(r, s), \operatorname{Control}(r, s)$ and $r e a(a, r)$ with $r, s \in A R$ and $a \in A g$, therefore encoding the descriptions of organizational structures in propositional logic (clauses, 2-5 Definition 3). The semantics of $O_{a}, G_{a}, K_{a}$ and $[\alpha(a)]$ operators and of the $D O N E(\alpha(a))$ (with $\alpha(a) \in \mathcal{A}$ ) assertions will then be given on the basis of standard Kripke models (clauses 8-12, Definition 3 ). 
To model organizational structures we make essentially use of the theory of directed graphs. However, we introduce only some basic elements of it, which are strictly of use for the development of the paper ${ }^{4}$.

\section{Definition 2 (Organizational structures)}

An organizational structure $O S$ is a tuple:

$$
\left\langle\text { Roles } \cup \text { Agents, } R_{\text {Power }}, R_{\text {Coordination }}, R_{\text {Control }}, \text { Rea }\right\rangle
$$

where Roles $\cup$ Agents is the finite set of roles and agents, and $R_{\text {Power }}, R_{\text {Coordination }}, R_{\text {Control }}$ are three irreflexive binary relations on Roles characterizing the Power, Coordination and Control structures. Rea is a subset of Agents $\times$ Roles and indicates which agents play which roles.

The following standard notions will be of use. An $R_{k}$-path (of length $n$ ) is a sequence $\left\langle x_{1}, \ldots, x_{n+1}\right\rangle$ of distinct elements of Roles s.t. $\forall x_{i} 1 \leq i \leq n, R_{k}\left(x_{i}, x_{i+1}\right)$. An $R_{k}$-semipath (of length $n$ ) is a sequence $\left\langle x_{1}, \ldots, x_{n+1}\right\rangle$ of distinct elements of Roles s.t. $\forall x_{i} 1 \leq i \leq n, R_{k}\left(x_{i}, x_{i+1}\right)$ or $R_{k}\left(x_{i+1}, x_{i}\right)$. A source in Roles is an element $s$ s.t. $\forall d \in$ Roles with $d \neq s$ there exists a $R_{k}$-path from $s$ to $d$. The indegree $i d_{k}(d)$ of a point $d$ in structure $k$ is the number of elements $d_{1}$ s.t. $R_{k}\left(d_{1}, d\right)$. The outdegree $\operatorname{od}_{k}(d)$ of a point $d$ in structure $k$ is the number of elements $d_{1}$ s.t. $R_{k}\left(d, d_{1}\right)$.

The semantics of $\mathrm{Org}$ can now be defined in terms of the following Kripke models.

Definition 3 (Semantics of $\mathrm{Org}$ )

The Org model is a tuple:

$$
\left\langle O S, W,\left(\mathcal{K}_{i}\right)_{i \in A g},\left(\mathcal{G}_{i}\right)_{i \in A g},\left(\mathcal{O}_{i}\right)_{i \in A g},\left(R_{\alpha(a)}\right)_{\alpha(a) \in \mathcal{A}}, I, J\right\rangle
$$

where $O S$ is an organizational structure; $W$ is a set of possible worlds, $\left(\mathcal{K}_{i}\right)_{i \in A g}$ are equivalence relations on $W,\left(\mathcal{G}_{i}\right)_{i \in A g}$ and $\left(\mathcal{O}_{i}\right)_{i \in A g}$ are serial binary relations on $W ;\left(R_{\alpha(a)}\right)_{\alpha(a) \in \mathcal{A}}$ are binary relations on $W ; I: P_{0} \cup P_{r} \longrightarrow 2^{W}$ is a truth assignment mapping each atomic proposition (except propositions in $P_{s}$ which get an explicit semantics) to the set of worlds in which it is true and $J=\left\langle J_{a}, J_{r}\right\rangle$ where $J_{a}: A g \longrightarrow$ Agents, i.e., $J_{a}$ is a function that maps agent names into agents, and $J_{r}: A R \longrightarrow$ Roles, i.e., $J_{r}$ is a function that maps role names into the corresponding roles. The satisfaction relation is defined as follows.

1. $M, w \models p$ iff $w \in I(p)$

2. $M, w \models \operatorname{Power}(r, s)$ iff $R_{\text {Power }}(J(r), J(s))$

3. $M, w \models$ Coordination $(r, s)$ iff

$$
R_{\text {Coordination }}(J(r), J(s))
$$

4. $M, w \models$ Control $(r, s)$ iff $R_{\text {Control }}(J(r), J(s))$

5. $M, w \models \operatorname{rea}(a, r)$ iff Rea $(J(a), J(r))$

6. $M, w \models \neg \phi$ iff $M, w \not \models \phi$

${ }^{4}$ For comprehensive expositions we refer the reader to [17, 16, 24]. 
7. $M, w \models \phi \wedge \psi$ iff $M, w \models \phi$ or $M, w \mid=\psi$

8. $M, w \models K_{i} \phi$ iff for all $u \in \mathcal{K}_{i}(w), M, u=\phi$

9. $M, w \models G_{i} \phi$ iff for all $u \in \mathcal{G}_{i}(w), M, u \models \phi$

10. $M, w \models O_{i} \phi$ iff for all $u \in \mathcal{O}_{i}(w), M, u=\phi$

11. $M, w \models[\alpha(a)] \phi$ iff for all $u \in \mathcal{R}_{\alpha(a)}(w), M, u \models \phi$

12. $M, w \models D O N E(\alpha(a))$ iff there exists only one $u \in W$ such that $(u, w) \in$ $\mathcal{R}_{\alpha(a)}$

Let us spend a few words to clarify this satisfaction definition. Clauses 1, 6 and 7 are the standard boolean ones. Clauses 8-11 are standard clauses for the satisfaction of modal formulas. With respect to the axiomatization of modal operators we take a standard perspective. For the knowledge operators $\left(K_{i}\right)$ we assume the axiomatization characterizing $\mathbf{S 5}$, for goal $\left(G_{i}\right)$ and obligation operators $\left(O_{i}\right)$ we assume the axiomatization characterizing $\mathbf{K D}$, and finally for actions $([\alpha(a)])$ we assume the axiomatization characterizing $\mathbf{K}^{5}$. Clause 12 is also quite standard for representing paths of actions actually occurred (see also [15]).

Clauses 2-5 provide an explicit semantics for propositions denoting structural configurations and can be actually read as an encoding of dyadic relations. It is worth noticing that a straightforward consequence of this semantics consists in making the propositions denoting structural configurations true either in all the worlds of the Org model or in none, that is, an organizational structure either holds in all the worlds of the model or in none. This is in fact perfectly sound with a conception of organizational structure as a hard constraint, a sort of skeleton, of the MAS.

The special propositions of the form $\operatorname{norm}(r, \phi), \operatorname{obj}(r, \phi)$ and $\inf (r, \phi)$ represent instead which state descriptions are considered to be, respectively, norms, objectives and information of a specific role. Since we are not interested in describing reasoning about norms, objectives and knowledge at the role level, but only at the agent one, these propositions do not get any specific semantics ${ }^{6}$. Nevertheless it is reasonable to assume that, for all $a \in A g, r \in A R$ and $\phi \in L_{0}$ :

1. $\operatorname{rea}(a, r) \wedge \operatorname{norm}(r, \phi) \rightarrow K_{a} O_{a} \phi$

2. $r e a(a, r) \wedge o b j(r, \phi) \rightarrow G_{a} \phi$

3. $\operatorname{rea}(a, r) \wedge \inf (r, \phi) \rightarrow K_{a} \phi$.

That is to say, given that agent $a$ enacts role $r$ : if $\phi$ represents a norm of that role, then agent $a$ knows that obligation $\phi$ holds for it (notice that in $\mathbf{S 5}, \vdash K_{i} \phi \rightarrow \phi$ ); if $\phi$ represents an objective of that role, then $\phi$ is in the goal base of $a$; if $\phi$ represents information presupposed by that role, then $\phi$ is in the knowledge base of $a$.

\footnotetext{
${ }^{5}$ For an extensive exposition of these systems the reader is referred to [1].

${ }^{6} \mathrm{~A}$ natural way to give a more elaborated semantics to them would be to consider and interpret them as modal operators.
} 


\subsection{Delegating, informing and monitoring}

We have not yet further specified the behavior of the special actions of delegating, informing and monitoring. We require that these special actions obey the following accordance schemata, for all $a, b \in A g$ with $a \neq b, \phi \in L_{0}$ and $\alpha(b) \in \mathcal{A}$, expressing the relation between knowledge and action (see [22]):

$$
\begin{aligned}
& K_{a}([\text { delegate }(a, b, \phi)] \psi) \rightarrow[\text { delegate }(a, b, \phi)] K_{a} \psi \\
& K_{a}([\text { inform }(a, b, \phi)] \psi) \rightarrow[\text { inform }(a, b, \phi)] K_{a} \psi \\
& K_{a}([\text { monitor }(a, D O N E(\alpha(b)))] \psi) \\
& \rightarrow[\text { monitor }(a, D O N E(\alpha(b)))] K_{a} \psi .
\end{aligned}
$$

Intuitively, the schemata state that the organizational actions of delegating, informing, and monitoring, once executed by an agent, always determine the knowledge about their necessary effects which the agent expects. To use the terminology of [22], these actions are always according to plan for each agent.

The first axiom could be enforced through the semantics by adding the following constraint concerning the interplay between the accessibility relations of an Org model. For all $a, b \in A g$ with $a \neq b, \phi \in L_{0}$ and $\alpha(b) \in \mathcal{A}$ :

$$
\begin{aligned}
& \forall w_{1}, w_{2}, w_{3} \in W\left(\mathcal{K}_{a}\left(w_{1}, w_{2}\right) \& R_{\text {delegate }(a, b, \phi)}\left(w_{2}, w_{3}\right)\right. \\
& \left.\Rightarrow \exists w_{4} \in W\left(R_{\text {delegate }(a, b, \phi)}\left(w_{1}, w_{4}\right) \& \mathcal{K}_{a}\left(w_{4}, w_{3}\right)\right)\right) .
\end{aligned}
$$

Perfectly analogous semantic constraints can be devised for the informing and monitoring action expressions.

We also assume that actors are always endowed with knowledge about all the necessary effects of the organizational actions they can perform. That is, for all $a, b \in A g$ with $a \neq b, \phi \in L_{0}$ and $\alpha(b) \in \mathcal{A}$, the following are to be considered axioms:

$$
\begin{aligned}
& {[\text { delegate }(a, b, \phi)] \psi \rightarrow K_{a}[\text { delegate }(a, b, \phi)] \psi} \\
& {[\text { inform }(a, b, \phi)] \psi \rightarrow K_{a}[\text { inform }(a, b, \phi)] \psi} \\
& {[\text { monitor }(a, D O N E(\alpha(b))] \psi} \\
& \rightarrow K_{a}[\text { monitor }(a, D O N E(\alpha(b))] \psi .
\end{aligned}
$$

Semantically, the first axiom is validated by the following relational constraint. For all $a, b \in A g$ with $a \neq b, \phi \in L_{0}$ and $\alpha(b) \in \mathcal{A}$ :

$$
\begin{aligned}
& \forall w_{1}, w_{2} \in W\left(R_{\text {delegate }(a, b, \phi)}\left(w_{1}, w_{2}\right)\right. \\
& \left.\Rightarrow \forall w_{3} \in W\left(\mathcal{K}_{a}\left(w_{1}, w_{3}\right) \Rightarrow R_{\text {delegate }(a, b, \phi)}\left(w_{3}, w_{2}\right)\right)\right) .
\end{aligned}
$$

Again, analogous constraints can be devised for the informing and monitoring action expressions.

To formally characterize the special actions in accordance with our intuitions about them, we need also to specify their pre- and postconditions. We consider the delegation and inform actions as having necessary preconditions, while the monitoring actions as having a guaranteed postcondition. This can be expressed as follows: 
Definition 4 (Delegate, inform, monitor)

For all $a, b \in$ Ag s.t. $a \neq b$ and $\phi \in L_{0}$ and $\alpha(b) \in \mathcal{A}$ :

$$
\begin{array}{ll}
- & \neg G_{a} \phi \rightarrow[\text { delegate }(a, b, \phi)] \perp \\
- & \neg K_{a} \phi \rightarrow[\operatorname{inform}(a, b, \phi)] \perp \\
- & {[\text { monitor }(a, D O N E(\alpha(b)))]\left(K_{a} D O N E(\alpha(b))\right.} \\
& \left.\vee K_{a} \neg D O N E(\alpha(b))\right) \\
- & \gamma \in L_{0}: \gamma \rightarrow[\operatorname{delegate}(a, b, \phi)] \gamma \\
- & \gamma \in L_{0}: \gamma \rightarrow[\operatorname{inform}(a, b, \phi)] \gamma \\
- & \gamma \in L_{0}: \gamma \rightarrow[\text { monitor }(a, D O N E(\alpha(b))] \gamma .
\end{array}
$$

Intuitively, in order to delegate a goal it is necessary to have it, but no specific consequence follows from a delegation. Analogously, to inform it is necessary to know, but the information does not necessarily create knowledge in the recipient actor. To monitor means to check (and know after that) whether another agent has done an action or not, that is to say, to monitor is an informative action ([22]) for the monitoring agent with respect to formulas concerning the performance of some action by some other agents. Finally, the last three schemata are a simplified formulation of frame axioms for the special actions, indicating that those actions only modify those parts of the world which concerns agents' obligations, goals, and knowledge.

The above specification of the pre- and postconditions of the special actions shows their effects without presuming an organization structure. In the next section we analyze the added value of structure showing that by adding the existence of an appropriate organization relation to the preconditions of these actions the effects of the actions increase, thus characterizing in what the structure precisely results.

\section{Modeling Organizational Structure}

\subsection{Variety of Structural Relations}

We characterize the power, coordination and control structural relations as follows:

Definition 5 (Power, coordination, control)

For all $a, b \in A g$ s.t. $a \neq b, \phi \in L_{0}$ and $\alpha(b) \in \mathcal{A}$ :

$$
\begin{aligned}
& (\operatorname{Power}(r, s) \wedge \operatorname{rea}(a, r) \wedge \operatorname{rea}(b, s)) \rightarrow[\text { delegate }(a, b, \phi)] O_{b} \phi \\
& (\text { Coordination }(r, s) \wedge \operatorname{rea}(a, r) \wedge \operatorname{rea}(b, s)) \rightarrow[\operatorname{inform}(a, b, \phi)] K_{b} \phi \\
& (\text { Control }(r, s) \wedge \operatorname{rea}(a, r) \wedge \operatorname{rea}(b, s) \wedge[\alpha(b)] \phi) \\
& \rightarrow\left[\text { monitor }(a, D O N E(\alpha(b))]\left(\neg \phi \rightarrow O_{a} \phi\right)\right. \\
& \left(\text { Control }(r, s) \wedge \operatorname{rea}(a, r) \wedge \operatorname{rea}(b, s) \wedge[\alpha(b)] \phi \wedge K_{a}\left(O_{b} \phi\right)\right) \\
& \rightarrow O_{a}(D O N E(\text { monitor }(a, D O N E(\alpha(b))))) .
\end{aligned}
$$


We have thus that:

- If a power relation exists between roles that are enacted by two agents then a delegate action has as effect an obligation for the recipient, that is, a delegate action implements then a form of "your wish is my command" principle.

- If a coordination link exists then the inform action automatically leads to an epistemic state in the recipient, the content of which consists in what has been communicated.

- If a control relation exists then the monitor action has as further consequence the generation of an obligation for the controller to achieve the state which the controlled actor was supposed to bring about. However, we consider the control relation to have also a normative aspect in that if an agent has control over another agent, it is then obliged to monitor it whenever it knows it has an obligation.

Some remarks are necessary. First, notice that we do not provide definitions of the relations, but just some consequences. Our aim is to capture some essential traits of those notions rather than providing a fully-fledged treatment of them. Different accounts of the phenomena of power, coordination and control are possible and would not harm the generality of the core thesis of our approach, i.e., organizational structure is multiple and enhances the effects, i.e., the postconditions, of agents' activities.

Secondly, notice that we understand those relations essentially as guaranteeing some effects to the basic actions of delegate, inform and monitor. Intuitively, if a power relation holds between roles $r$ and $s$, all delegation acts performed by an agent $a$ enacting role $r$ on agents enacting role $s$ succeed in creating an obligation for these agents. Analogously, if a coordination relation holds between roles $r$ and $s$, all information acts performed by agents enacting role $r$ to agents enacting role $s$ are successful in the sense that they create knowledge in these agents. Notice that this is only one way of interpreting this relation based on considering coordination essentially as "the process of managing interdependencies between activities" ([7]) with respect only to the flow of knowledge between agents. The relation can alternatively get a normative flavor, expressing that agents should inform another agent if they are connected through a coordination link. Finally, if a control relation holds between roles $r$ and $s$, all monitoring acts performed by agents enacting role $r$ on agents enacting role $s$ do not only create knowledge in the controller about the relevant state of affairs (see Definition 4), but they also determine an obligation for the controller in case the controlled agent did not perform the action that is monitored. This indicates some kind of taking responsibility for a failure.

\subsection{Properties of Structures}

This formal characterization of the three basic dimensions of structure in organizations allows us to give a concrete and rigorous meaning to some simple properties of this structure. In what follows some properties of digraphs are listed and an intuitive interpretation of them in terms of Definition 2 and Definition 5 is provided. 
Existence of an $R_{k}$-path between two elements. A $R_{\text {Power-path between } r \text { and }}$ $s$ of length $n>1$ determines the possibility, for the intermediate actors, to delegate delegated goals. Analogously, a $R_{\text {Coordination }}$-path between $r$ and $s$ of length $n>1$ determines the possibility, for the intermediate actors, to transmit the received information. If the coordination structure is strongly connected (i.e. there is a path between any two points in the graph) then information can be shared between all agents in the organization. For power and coordination, the shorter the path the quicker and the more efficient is the generation of obligations and the transmission of knowledge. A $R_{\text {Control }}$-path between $r$ and $s$ of length $n>1$, instead, corresponds to a sort of control of the controller chain. In this case, the longer the path, the tighter can be the control.

Existence of a source. In a power structure the existence of a unique source for the $R_{\text {Power }}$ relation would express the so-called unity of command principle or, to use the terminology of [23], the existence of a highest competence, that is, a sort of source (ideal or embodied) of all tasks of the organization. The length of the $R_{\text {Power }}{ }^{-}$ paths emanating from the source gives the measure of the distance of each element from the source. If the power structure is intransitive, the existence of a unique source formalizes the idea of a pyramidal shape of the organization, also known as scalar principle. This principle is not implemented in organizations in which more sources for the $R_{\text {Power }}$ relation exist. In this case, symmetric $R_{\text {Power }}$ links connect the sources determining the absence of a highest single competence, but, on the other hand, the presence of a cluster of roles forming a sort of oligarchy. A source for the $R_{\text {Coordination }}$ relation can be seen as a form of transmission point for the whole organization. In this case, a unique source is obviously not recommendable, it implying the absence of any feedback (symmetric link) toward the source itself. Such a property would instead be less undesirable for the control structure, it implying the existence of an actor which either can monitor everything or can monitor the performance of all controlling actors of the organization.

Indegree of an element equal to 1 . In a power structure, an element $d$ having $\operatorname{id}_{\text {Power }}=1$ would represent a role to which only one superordinate role can delegate tasks. If the indegree of all the elements that are not the source is 1, then commands flow through the power structure according to what is usually called, in organization theory, an unambiguous chains of commands. If the indegree is higher than 1, the delegation can give rise to redundancies and ambiguities, generating the possibility of authority conflicts, as formally described already in [13]. On the other hand, ambiguity can happen to be useful in cases in which more flexibility is required, so that the chain of command can remain intact despite the loss of an actor. Analogously, redundancies are particularly useful from the point of view of a coordination structure, where a multiplicity of communication channels is often recommended, and of a control structure, where the more are the controllers, the more efficiently implemented is the monitoring activity.

Outdegree of an element equal to 1 . In a power structure, an element $d$ having $\operatorname{od}_{\text {Power }}=1$ denotes an element with low direct power. Notice that a source in the structure, though occupying a position from which it can reach every other element, if it has outdegree 1 it has direct power only on the first subordinated element (to which

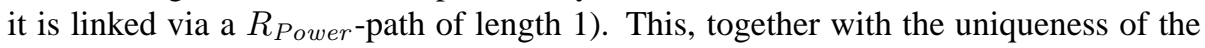
source, corresponds to a quite standard vision of the so-called "hierarchies". Interest- 
ingly, indegree and outdegree are strictly related with the transitivity or intransitivity of the relation at issue. For example, in a transitive "hierarchy", the source can reach everybody directly and indirectly; as a drawback, every point, except the source itself and those linked to it via an $R_{\text {Power }}$-path of length 1 , are in a position of multiple authority conflicts. Moreover, in this case, the scalar principle is not met. With respect to $R_{\text {Power }}$ then, the choice for low outdegrees appear to be a sensible structural solution. Coming to coordination and control structures instead, higher outdegrees make more sense since elements with outdegree higher than one denote that there are roles which have a high communication possibilities and, respectively, which control many different roles.

With this informal discussion we intended to show how basic concepts of graph theory can be usefully imported and meaningfully interpreted in terms of organization theory, therefore showing the usefulness of rigorous models of social structure such as the one proposed in Definition 2 and Definition 5 on which our framework is based. On this ground, formally specified taxonomies for each dimension of organizational structure could be systematically worked out and analyzed.

A further aspect needs though to be investigated, namely how the three dimensions of power, coordination and control interact, and how they should interact in soundly specified organization. A definition of sound organizational structure constitutes the basic result of the following section.

\subsection{Structural Interplay}

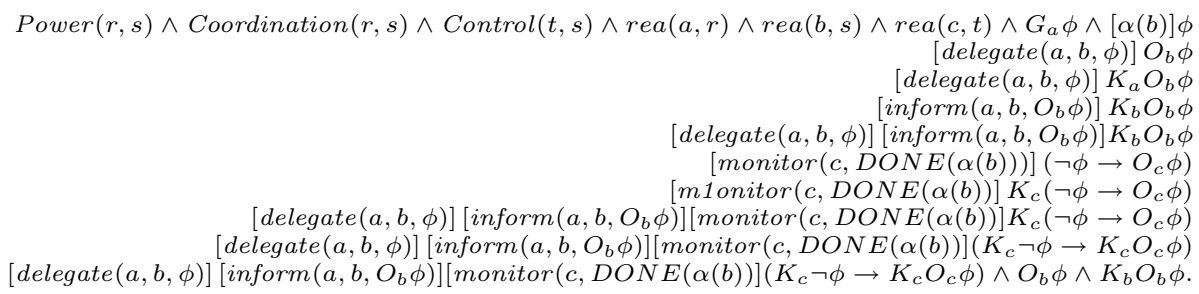

Figure 1: Deriving the effect of structure

In the previous sections we discussed aspects that appear to be relevant for each of the structural dimensions considered in isolation: first with respect to their effects on agents' activities (Section 4.1) and then with respect to their inherent structural properties (Section 4.2). In this section we analyze how the different dimensions might interact.

Structure is necessary, first of all, in order to reassign goals via new obligations (delegation). Because of this dynamics organizations need to distribute relevant knowledge (information), and implement forms of performance assessment and recovery (monitoring). Somehow, the interplay between these structural dimensions lies in the delegation activity and is therefore based on the power relation. This is in perfect accordance with many foundational investigations in the theory of organizations ([25, 23]). In particular: 
delegation is the primordial organizational act, a precarious venture which requires the continuous elaboration of formal mechanisms of coordination and control ([25]).

This observation can be distilled in the following two principles: organization structure should see to it that each agent is always aware of its duties (an "ought implies know" principle); organization structure should see to it that its objectives are met (a "successful performance" principle). They can be expressed, in Org, as follows. For all $a \in A g, r \in A R$ and $\phi \in L_{0}$ :

$$
\begin{aligned}
\text { ought implies know } & O_{a} \phi \rightarrow K_{a} O_{a} \phi \\
\text { successful performance } & o b j(r, \phi) \rightarrow \bigvee_{a \in A g}\left(G_{a} \phi \vee O_{a} \phi\right)
\end{aligned}
$$

It is easy to see that the implementation of the "ought implies know" can be met by aligning the coordination structure with the power structure. The successful performance can never be guaranteed as the agents are autonomous and subject to failure and thus can always fail to meet their goals and/or obligations. The control structure cannot guarantee a full implementation of the "successful performance" principle. The effect of a monitor action in a position of control is a new obligation for the controller if the goal has not been achieved. In principle, also the controller can then violate this obligation leaving the goal not achieved. This might be considered an undesirable property, since it leaves the organization constitutively open to unsuccessful performances: in other words the very result of the control structure is to guarantee the accomplishment of goals, or to further motivate that accomplishment via norms. Nevertheless, this feature faithfully mirrors the nature of human organizations where control and recovery activities are also activities which are open to failure, and where the problem of the control of the controllers is an unsolvable issue. This explains also why the principle of "successful performance" can therefore be implemented only at some degree through adding iterated levels of control. The more levels of controls are enacted, the stronger the principle can be thought of being implemented: Control $(a, b)$, Control $(c, b)$, Control $(d, c)$, and so on, exactly as it happens in human organizations.

These remarks are formally illustrated in Figure 1. The derivation is explained line by line in what follows: (1) is assumed; (2) is obtained via (1) Definition 5 and MP; (3) via (2), Necessitation, Accordance and MP; (4) via (1), Definition 5 and MP; (5) via (4) and Necessitation; (6) via (1), Definition 5 and MP; (7) via (6), Necessitation, Accordance and MP; (8) via (7) and Necessitation; (9) via (8) and Distribution; finally, (10) via (9) and Definition 4.

In Figure 1 is then displayed a derivation of the effect of the simplest structural configuration: coordination and control guarantee that the inform and monitor actions efficiently contribute to the achievement of the delegated goals. The last line of the derivation can be seen as displaying the basic organizational performance unit: a delegation action followed by at least one information action and by at least one monitoring action.

From this derivation we see that if we want to guarantee to some extent that after a delegation through a power relation the delegated action is actually performed, we should take care that the agent that is obliged to perform the action also knows about 
this. This can only be ensured through a successful inform action to that agent through a coordination link that is either direct or indirect. If we also want to have a back-up in case of failure we should make sure that there is an agent monitoring the delegated action through a control link.

As a result, our analysis delivers a representation of organizations as triple structures. If we want the organization to adhere to some intuitive properties they should be constrained in order to meet precise structural principles concerning the interplay between power, coordination and control. This leads us to the following definition of sound organizations which extends Definition 2.

\section{Definition 6 (Sound Organizations)}

A sound organization is a tuple:

$$
\left\langle\text { Roles } \cup \text { Agents, } R_{\text {Power }}, R_{\text {Coordination }}, R_{\text {Control }}, \text { Rea }\right\rangle
$$

where Roles $\cup$ Agents is the finite set of roles and agents, Rea $\subseteq$ Agents $\times$ Roles, and $R_{\text {Power }}, R_{\text {Coordination }}, R_{\text {Control }}$ are three irreflexive binary relations on Roles such that $\forall r, s \in$ Roles:

$$
\begin{aligned}
R_{\text {Power }}(r, s) \Rightarrow & \text { there exists a } R_{\text {Coordination }}-\text { path } \\
& \text { from r to } s ; \\
R_{\text {Power }}(r, s) \Rightarrow & \text { there exists a } t \in \text { Roles } \\
& \text { s.t. } R_{\text {Control }}(t, s) .
\end{aligned}
$$

The occurrence of a power relation between role $r$ and role $s$ requires: first, in order for the structural principle "ought implies know" to be met, the existence of a (finite) coordination path from $r$ to $s$ so that effective inform actions can transmit the relevant knowledge (essentially about obligations) of agents enacting role $r$ to agents enacting role $s$; second, in order for the structural principle "successful performance" to (try to) be met, the existence of at least an element $t$ (which, notice, might be $r$ itself) which is in a control relation with $s$.

\section{Conclusions}

In this work we argued for two central theses. The first thesis consisted in holding that organizational structures exhibit at least three relevant dimensions, namely the dimensions of power, delegation and control and not just one. In addition, these three dimensions have been also shown to need to be interrelated in precise ways in order to give rise to sensibly designed organizational structures. In a nutshell, we therefore viewed organizations as multi-structured objects. The second thesis consisted in considering the effect of organizational structure on the agents involved in an organization specifically as what enhances the effects of agents' activities such as delegating, informing and monitoring. On the basis of these theses, we developed a multi-modal logic framework able to represent structural configurations and reason about (some of) their effects on agents. 
Future work will be focused first of all on considering more structural dimensions (such as the "empowering" dimension informally touched upon in Section 2) and on improving Definition 4 and Definition 5 in order to enable more detailed and elaborated characterizations of the effects of organizational structures.

\section{References}

[1] P. Blackburn, M. de Rijke, and Y. Venema. Modal Logic. Cambridge University Press, Cambridge, 2001.

[2] C. Castelfranchi. The micro-macro constitution of power. ProtoSociology, 18:208-268, 2003.

[3] C. Castelfranchi and R. Falcone. From task delegation to role delegation. In M. Lenzerini, editor, LNAI 1321. AI*IA 97: Advances in Artificial Intelligence, pages 278-289. Springer Verlag, Berlin, 1997.

[4] M. Dastani, V. Dignum, and F. Dignum. Organizations and normative agents. In Proceedings of the First Eurasian Conference on Advances in Information and Communication Technology (EurAsia-ICT 2002) Tehran, Iran, 2002.

[5] M. Dastani, V. Dignum, and F. Dignum. Role-assignment in open agent societies. In Proceedings of the Second International Conference on Autonomous Agents and Multiagent Systems (AAMAS'03), Melbourne, July. ACM Press, 2003.

[6] M. Dastani, B. van Riemsdijk, F. Dignum, and J.-J. C. Meyer. Enacting and deacting roles in agent programming. In Proceedings of the 5th International Workshop on Agent-Oriented Software Engineering (AOSE'04), 2004.

[7] K. Decker and V. Lesser. Designing a family of coordination algorithms. In V. Lesser, editor, Proceedings of ICMAS'95, pages 73-80, San Francisco, CA, USA, 1995. The MIT Press: Cambridge, MA, USA.

[8] F. Dignum. Autonomous agents with norms. Artificial Intelligenc and law, 7:6979, 1999.

[9] V. Dignum. A Model for Organizational Interaction. SIKS Dissertation Series, 2003.

[10] V. Dignum, J.-J. C. Meyer, H. Weigand, and F. Dignum. An organizationaloriented model for agent societies. In Proceedings of International Workshop on Regulated Agent-Based Social Systems: Theories and Applications (RASTA'02), at AAMAS, Bologna, Italy, 2002.

[11] P. Doreian (ed.). Journal of mathematical sociology. Taylor \& Francis.

[12] T. J. Fararo. Reflections on mathematical sociology. Sociological Forum, 12:73$101,1997$. 
[13] M. F. Friedell. Organizations as semilattices. American Sociological Review, 32:46-54, 1967.

[14] A. Giddens. Social Theory and Modern Sociology. Polity Press, 1984.

[15] D. Grossi, F. Dignum, L. Royakkers, and J.-J. C. Meyer. Collective obligations and agents: Who gets the blame? In A. Lomuscio and D. Nute, editors, Proceedings of DEON'04, pages 129-145, 2004.

[16] F. Harary. Graph Theory. Addison-Wesley, London, 1969.

[17] F. Harary, R. Norman, and D. Cartwright. Structural Models: An Introduction to the Theory of Directed Graphs. John Wiley \& Sons, New York, 1965.

[18] D. Harel. Dynamic logic. In D. Gabbay and F. Guenthner, editors, Handbook of Philosophical Logic: Volume II: Extensions of Classical Logic, pages 497-604. Reidel, Dordrecht, 1984.

[19] B. Horling and V. Lesser. A Survey of Multi-Agent Organizational Paradigms. Computer Science Technical Report 04-45, University of Massachusetts, May 2004.

[20] T. Ioerger and C. Johnson. A formal model of responsibilities in agent-based teamwork. In Proceedings of the fifth international conference on Autonomous Agents, Montreal, Canada. ACM Press, 2001.

[21] A. J. I. Jones and M. Sergot. A formal characterization of institutionalised power. Journal of the IGPL, 3:429-445, 1996.

[22] J.-J. Meyer and W. van der Hoek. Epistemic Logic for AI and Computer Science, volume 41 of Cambridge Tracts in Theoretical Computer Science. Cambridge University Press, 1995. 25.5.103.

[23] O. Morgenstern. Prolegomena to a theory of organizations. Manuscript, 1951.

[24] K. A. Ross and C. R. B. Wright. Graph Theory. Prentice-Hall, New Jersey, 1992.

[25] P. Selznick. Foundations of the theory of organization. American Sociological Review, 13:25-35, 1948.

[26] A. B. Sørensen. Mathematical models in sociology. Annual Review of Sociology, 4:345-371, 1978. 